\title{
STRENGTHENING STUdENT KNOWLEDGE THROUGH INSTRUCTIONAL VIDEOS OF HANDS-ON ACTIVITIES
}

\author{
Chloe Gibson, Kayleanna Giesinger, Rania Al-Hammoud and Wayne Broadland \\ University of Waterloo \\ chloe.j.gibson@edu.uwaterloo.ca,kgiesing@edu.uwaterloo.ca, ralhammo@uwaterloo.ca, \\ brodland@uwaterloo.ca
}

\begin{abstract}
The use of hands-on activities has been proven in the past to be effective in teaching pedagogies. Recognizing this need, a first year Mechanics course at the University of Waterloo has already implemented the use of seven hands-on activities. Instructors of the course have found certain time limitations which results in students only participating in two of the seven activities. To continue improving student learning, instructional videos were developed to solve this problem. The techniques used for video development incorporate learning pedagogies to foster deeper learning throughout the viewing experience. These techniques include simulating experiential learning and reflective learning. In each video, a breakdown of the activity building and experimenting process is demonstrated. This is done through people physically interacting with the models as students would in the classroom. Accompanying the demonstration is an illustration of various mistakes students often make during the activities. Errors are discussed, and their outcomes are shown using course concepts to reinforce the appropriate processes. In addition, questions are posed to the viewers throughout each of the videos.
\end{abstract}

Keywords: Experiential, Models, Videos, knowledge retention, deep learning.

\section{INTRODUCTION}

Mechanics is a core first-year course on which 5 to 15 other courses build, depending on a student's program of study. Many of the concepts in the course are counter intuitive which makes the use of physical models essential to learning. While it is ideal for students to handle the models, carefully scripted videos could allow them to experience them vicariously, engage the key ideas deeply, and reflect on the challenging ideas they demonstrate. A first year Mechanics course at the University of Waterloo has already implemented the use of seven hands-on activities. Due to time limitations, students are only able to participate in two of the seven activities. Recognizing the benefits of using video media as a learning platform, instructional videos were developed to allow students to experience the videos they could not participate in.

The intent of the video development was to provide a resource for students to vicariously "experience" the activities that they did not have time to do. Each video was carefully planned to incorporate the essence of doing the hands-on activities. This is done by highlighting central concepts, according to the educational objectives of each separate model. Key steps in the activities will be filmed and accompanied by explanations that drive home the fundamental concepts. The models are very "physical" and lend themselves well to filming and to demonstrating behaviors the students would otherwise probably not really believe. This allows for the videos to incorporate the vital deeper learning techniques of the activities.

The videos are also aiming to be a complimentary learning resource to the activities. Viewing the videos before and after completing the activities will improve efficiency, allow for more thoughtful reflection and serve as a learning tool for students to review. Students can view the videos associated with their assigned activities before doing those activities. Cooper et al. (2015), stated that, "learning by observing and imitating others has long been recognized as one of the most basic yet powerful learning strategies for humans." Students cannot always see the overall concept until the end result is revealed. This causes students to be slower and more inefficient with their time since they are trying to understand the steps rather than the concept. In a study, Wynkoop (2015) found that when students used a video as instruction they required less time to complete their task. By watching the videos prior to doing the activity students will better carry out the activities within the 50 minute length of their tutorials. The video will give them awareness of the tasks they will do and will help them to focus on its key steps and concepts. It will thus help them to be better prepared and more efficient in completing the required tasks. 
Additionally, the counter intuitive concepts of the mechanics course can be better reflected on with the use of the videos. Through reflection understanding can settle in, take root and displace previously held misconceptions. The videos will be structured to identify and drive home these often-challenging ideas. The videos will be structured to identify and drive home these oftenchallenging ideas. Currently, students do not have the opportunity to observe the activities before doing them. This eliminates any potential initial reflective learning and limits experiential learning. De Boer et al. (2016) state that students need prior knowledge at a certain minimum threshold in order to expand their knowledge network in long term memory. By watching the videos, students gain initial knowledge

of

the activity, enabling them to form relevant questions and make predictions based on the viewed material. Sherin (2004) reinforces this by stating that students who have prior exposure to materials have, "the opportunity to develop a different kind of knowledge for teachingknowledge not of 'what to do next,' but rather, knowledge of how to interpret and reflect on classroom practices". Therefore, by being exposed to the material students can have a continual reflection process throughout the activity, which will then improve their decision making and problem solving skills within the activities.

Separate from the activity the videos can be an effective source for studying. The videos will be structured to cover fundamental mechanics concepts in an engaging way. Thus, they can serve as a study tool, a way to review key ideas on demand and a means to repetitively engage difficult ideas. Mayer et al. (2002) find instructional videos to be a comparable alternative to in-class instruction when class time is limited. Likewise, Anderson (2008) states that online study resources, "provide efficient and timely access to learning materials", allowing for students to learn in their preferred time and environment. Having videos available to the students allows for them to explore their future concepts in a time that best suits their individual educational needs. When studying students often search for a video on a specific topic and settle for one that is close to their educational needs. The development of videos for our custom class activities would provide a targeted resource for student study needs. We expect students across many of our engineering programs and in different years will view the videos online. We hope that they will be viewed by students around the world.

\section{VIDEO DEVELOPMENT}

A series of ten videos focusing on a range of mechanics topics were conceptualized and produced by a team possessing a variety of skills and perspectives with the goal to provide videos for students that would engage and deepen their understanding of mechanics. The developing process was largely informed by the work put into creating the first video of the series. That video was constantly reworked, had every aspect discussed in detail, and was questioned at every step, which gave rise to a final product that shaped the basic structure and main elements present in each of the following videos. The methods employed to produce this initial video brought about a general procedure that would be followed to ensure efficiency and success in the process of developing the series. There was flexibility in the structure and timeline for production that came from the natural flow of ideas and the understanding that certain concepts required more attention than others. Yet, there were also several steps that remained constant in producing each video. One of these steps was to outline a high-level overview of the related activity. The team would go through the activity steps and identify its core learning objectives, determine its key insights and assessment points, and list common errors student make in understanding the concepts. This information proved valuable when forming a first draft, as it largely helped to shape the script and accompanying storyboards. Upon completion of the first draft, the project team would hold a meeting to review it thoroughly and discuss ideas. The discussion would often involve suggesting examples to include, questions to ask and how to phrase them, and images or video clips that would best work to complement the narration. The goal of the meeting was also to determine any aspects that could be improved upon by identifying points of confusion due to over or under explanation, words likely uncommon to students, where definitions should be included, and whether certain ideas were feasible. Any notes generated from the meeting would be used to make changes to the script and storyboard. Generally, the following stage in the process would be the pre-production meeting, which took place after the proposed changes were included and the script was near completion. The goal of the meeting was to finalize everything prior to the start of filming. Primarily, it was used to determine the logistics of filming, identify any final modifications to the storyboards, and confirm the details of the aminations. Soon after, the necessary filming would take place, followed by the recording of the narration, the editing of the film, and the addition of the animations. Finally, everything would be reviewed for any final changes, and once all were satisfied with the result, the video was deemed complete. After all ten videos had been finalized, they were made available for anyone to view (see Appendix for a complete list of the videos with links to watch them online).

One of the main concerns with attempting to recreate the classroom activities through a series of videos was that the many benefits that come with having hands-on experience with the models would be lost. To make up for 
the lack of real world interaction on the part of the viewer, one of the focuses early on was to determine alternative ways to provide an experience comparable to the activity. Capturing the essence of participating in the classroom was achieved in part by using the activity instructions as the starting point for each video. Prior to commencing any work on a video, the entire team would go through the equivalent model activity to get a true feel of what a student experiences in the classroom. Additionally, during the writing and planning phase, the activity steps were placed alongside the corresponding storyboards to strengthen the connections between the two and ensure that significant steps from the activities were incorporated into the video. To explore the more experimental nature of the activities, it was important that the videos illustrated mistakes made by students when first working with the models. At several points over the course of the videos, the models are assembled and used in such a way that results in the collapse or unwanted movement of their components. A few incorrect attempts would be made, followed by a demonstration of the correct execution along with a brief explanation of why certain methods proved unsuccessful. The faults incorporated into the narratives were those found to be regularly observed in the classroom and proved essential in reinforcing the key concepts. A few examples of the errors depicted in the videos include using the wrong materials, implementing an improper construction order, and incorrectly pairing the model components. While all these strategies were helpful, depicting every aspect of the activity process and how the models work would still fail to provide the viewer with any instinctual feeling or real connection to the concepts. As a solution to this problem, the videos incorporate other examples besides the models that are able to similarly illustrate the central ideas. Specifically, examples were selected that many would likely possess some memory of experiencing physically first-hand. The development process involved discussing various ideas for such examples with the goal to find one that was as universal as possible, involved a certain degree of physicality, and applied to the concept well. After much deliberation, examples were chosen and incorporated into the videos that are anticipated will provide viewers with a physical, gut feel for the concepts without requiring handson experience with the models.

In developing the videos, it was thought to be essential that they appeal to people possessing varying levels of familiarity with the concepts and models. In many ways, the videos cater to those without the opportunity to experience the models; however, the series was also designed with the knowledge that the videos would be watched by many students prior to, or after having gone through the related activity in the classroom. With these students in mind, the videos were viewed as an opportunity to improve the efficiency of the activities, and in turn allow for more of the allotted time to be used for experimentation and developing deeper connections with the material. Deciding to centre the focus of an activity on the overall process involved, rather than fixating on the end result, was a significant factor in achieving this goal. It included depicting both a thorough step by step building procedure, and a fair amount of experimentation with the models. Additionally, it involved the display of people physically interacting with the models as shown in Fig. 1. This further served to mirror the roles students are to take on in the classroom. Moreover, there were certain advantages to working with video as a medium that would allow for watching a video based on a given activity to be more beneficial to students than simply reading instructions or a brief description of that activity. One of these advantages was the use of video making it possible for the time dedicated to explaining each step to be determined based on that step's overall importance. That is, the videos could highlight the more complex, learning-focused steps of the activity, while spending only a few seconds on the basics in order to make efficient use of the students' time. Another valuable aspect of working with video is the ability to use varied methods to communicate an idea to the viewer. The videos employed narration, moving images, still images, graphical overlays, and text and these were coordinated to better reinforce points that were particularly significant or difficult to understand. A common use for this was to help familiarize students with objects or components from the model kits. Where uncommon objects are introduced in the videos, an image of the object would appear onscreen, followed by text with an arrow or some other identifier linking the two, along with narration of the object's name and a short description of its significance. These scenes aid in acquainting students with the names of various uncommon model components, allowing them to navigate the activity more efficiently. Similarly, text was displayed onscreen as a supplement to the narration in order to highlight the significant ideas in a video and emphasize key terminology.

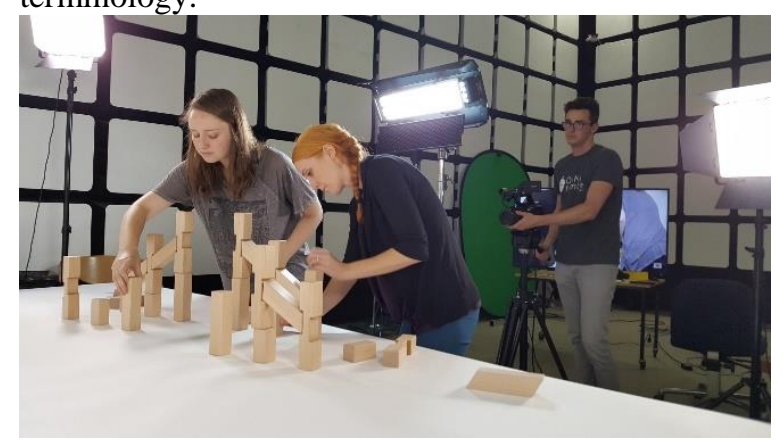

Figure 1 Assembling the model during the filming process

The videos were developed with the goal of going beyond simply presenting the viewer with information on the given topic, as they aim to engrain a deep understanding of the presented ideas. Developing the videos was largely centered on exploring methods to better engage students in the video, thus discouraging passive 
watching and leading them to better reflect on the core concepts being discussed. The primary means through which they encourage reflection is posing questions to the viewer at multiple instances throughout each video. The questions are asked before a new concept is introduced or any in-depth explanation is provided on the topic. Each is expressed verbally through the narration and visually with accompanying text that appears onscreen. This is followed by a pause of three seconds, along with a prompt for the viewer to pause the video and contemplate their response. These strategies were employed to encourage viewers to reflect on the question and give them sufficient time to reach a conclusion. Each question was thoughtfully considered in terms of its clarity, difficulty, likely responses, and relation to the main concepts. They were incorporated into the series with the goal to inspire viewers to become more engaged in the subject matter. An additional purpose of the questions is to prompt viewers to reflect on their prior knowledge about the topic or acknowledge any instincts they may have relating to its application. A few general examples of questions incorporated into the videos include asking the viewer what will happen when a certain action is performed, why a certain result was observed, what order to construct the model, whether a failure will occur for a given scenario, and which of two options is the better design. After being posed, the answer is revealed along with a short explanation on why it is the correct response. People who come to an incorrect conclusion are able to understand the error in their thinking, while for those who answered correctly, it reinforces what they already know and instills confidence in their knowledge. Reflection is further encouraged through the interconnectedness of the videos in terms of the concepts discussed and how they are presented. Viewers who watch multiple videos from the series may have the opportunity to reflect on knowledge gained from a video they watched previously to better understand a related topic presented in a later video.

Throughout the video development process, the team was highly aware of the value students could find in using the videos as a study tool. Maximizing the use of the videos for students to supplement their review of course material largely influenced the structure and content of the videos. Each video was outlined to follow a logical order and have similar ideas grouped to allow viewers to easily find and re-watch a specific concept they may be struggling with. The length of the video was another key factor considered, as the goal was to adequately cover each of the main concepts with the least amount of time. The team anticipated that this would encourage efficient use of study time and prevent the viewer from losing focus. Aiding students in their review of the material was further prioritized through the incorporation of questions. They could be used to assist students in judging their understanding of the topic and gain a better sense of the gaps in their knowledge.

\section{Acknowledgements}

First the Engineering Ideas Clinic is to be thanked for their resources and financial support. Next the University of Waterloo LITE Grant is acknowledged for their significant funding put toward the development of the videos

\section{References}

[1] Anderson, Terry. Theory and Practice of Online Learning. Edmonton: AU Press, 2008. Print.

[2] Cooper, Darren and Steve Higgins. "The Effectiveness of Online Instructional Videos in the Acquisition and Demonstration of Cognitive, Affective and Psychomotor Rehabilitation Skills". Br J Educ Technol 46.4 (2014): 768-779. Web.

[3] De Boer, Jelle, Kommers, Piet A. M., De Brock, Bert and Tolboom, Jos "The Influence of Prior Knowledge and Viewing Repertoire on Learning from Video". Educ Inf Technol $21.5 \quad$ (2014): 1135-1151.Web.

[4] Mayer, Richard E. and Roxana Moreno. "Aids to Computer-Based Multimedia Learning". Learning and Instruction 12.1 (2002): 107-119. Web.

[5] Sherin, M. G. (2004). New perspectives on the role of video in teacher education. In J. Brophy (Ed.), Advances in research on teaching. Vol. 10: Using video in teacher education (pp. 1-27). Oxford, UK:Elsevier.

[6] Wynkoop, K. S. "Watch This! A Guide to Implementing Video Modeling in the Classroom". Intervention in School and Clinic 51.3 (2015): 178-183. Web.

\section{APPENDIX A: LINKS TO ENGINEERING MODELS VIDEOS}

1. Equilibrium of a Point:

https://www.youtube.com/watch?v=a2IX52U1CWE

2. Equilibrium of a Body:

https://www.youtube.com/watch?v=6zXkYjmvLuI

3. Arches and Chains: 
Proc. 2018 Canadian Engineering Education Association (CEEA-ACEG18) Conf.

https://www.youtube.com/watch?v=JlL6ZHChhQE

4. Suspension Bridges:

https://www.youtube.com/watch?v=caTaBeKUh-U

5. Gothic Cathedrals:

https://www.youtube.com/watch?v=WiX2amY7G-I

6. Soil and Water Pressure:

https://www.youtube.com/watch?v=mpV8-YaJ2p4

7. Dams:
https://www.youtube.com/watch?v=NaVCKTYQEr4

8. Retaining Walls:

https://www.youtube.com/watch?v=iDzp6xEAT2I

9. Silos and Tanks:

https://www.youtube.com/watch?v=YZVIRILUkCc

10. Tunnels and Culverts:

https://www.youtube.com/watch?v=62-pK2ISlv4 